\title{
Who Will Do the Necessary Experiments?
}

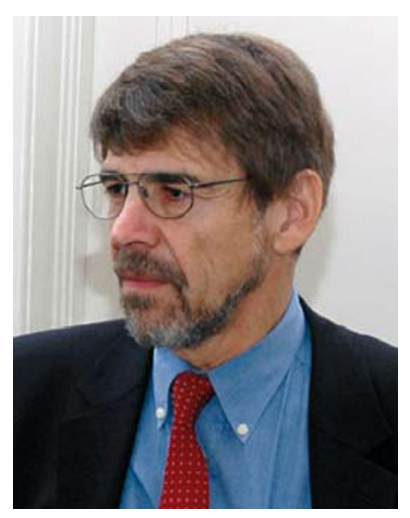

Herbert Ipser

Last year I attended the TOFA (Thermodynamics of Alloys) Meeting in Santos, Brazil. Quite a number of contributions were on phase diagram optimizations using the well-known CALPHAD method. As the CALPHAD method is a semi-empirical method, one needs a minimum of experimental data, both thermodynamic data as well as phase diagram information, in order to arrive at a reliable and consistent description of the binary or higher-order system. If experimental enthalpies of formation are not available, ab initio calculations may provide additional input into the CALPHAD calculations.

Several of the presenters ended their talk with the remark that additional experimental data would be necessary or, at least, helpful. And several times I was tempted to raise my hand in the discussion and ask why he or she never tried to do these necessary experiments themselves. Many of our younger colleagues are highly proficient in doing CALPHAD optimizations or even ab initio calculations, and still a considerable number are able to determine phase equilibria experimentally using $\mathrm{x}$-ray diffraction, scanning electron microscopy, and thermal analyses. However, the number of labs where thermodynamic properties of metallic or ceramic systems are measured is dwindling fast. There may be still a number of scientists who know how to use a calorimeter (although several of them are approaching or beyond their regular retirement age), but how many labs do still exist where vapor pressure or emf measurements are done, although these are valuable sources of (partial) Gibbs energy data?

Today we still rely on extremely accurate measurements from the middle of the last century. Just a few examples are the measurements of heat capacities in the low temperature range by Edgar F. Westrum at the University of Michigan, which were necessary to calculate standard entropy values; or the heat capacity measurements in the medium temperature range up to about $800 \mathrm{~K}$ by Frederik Grønvold at the University of Oslo; or the vapor pressure and Gibbs energy data determined by torsion effusion mass spectrometry in the labs of Gerd M. Rosenblatt at Princeton University or Jimmie G. Edwards at the University of Toledo (Ohio). All these labs, and many others, have been closed down, some of them long ago, and the equipment has been disassembled. But who will do such careful, but admittedly tedious, experiments in the future?

Of course, doing thermodynamic measurements is not really "sexy," and some people may consider these experiments awfully boring. But it is a fact that we do need more experimental data, be it enthalpies of formation of solid compounds or enthalpies of mixing of liquids, be it accurate heat capacities, or be it vapor pressure or emf data from which Gibbs energies can be derived! And from my own experience, it is not boring at all to do thermodynamic 
experiments. It is true, using a computer and appropriate software (in many cases simply as a black box) can provide results rather quickly, and on the other hand, it is more tedious and time consuming to run experiments where one has to wait for the results and needs to interpret and compare them continuously to see if they are consistent with earlier data within the same series of measurements. But at the end it is equally satisfactory to have a consistent and reliable set of results that may be valuable for future scientific work.
Thus I would like to encourage some of our younger colleagues to try it, to try to do thermodynamic experiments by themselves. I am sure they might like it after all.

\section{Herbert Ipser}

Associate Editor, Journal of Phase Equilibria and Diffusion

Institute of Inorganic Chemistry/Materials Chemistry University of Vienna 